\title{
FePd, FePt, and CoPt alloy epitaxial thin films with flat surface grown on $\mathrm{MgO}(111)$ substrate
}

\author{
Akira Itabashi ${ }^{1}$, Mitsuru Ohtake ${ }^{1}$, Shouhei Ouchi $^{1}$, Fumiyoshi Kirino ${ }^{2}$, and Masaaki Futamoto ${ }^{1, a}$ \\ ${ }^{1}$ Faculty of Science and Engineering, Chuo University, 1-13-27 Kasuga, Bunkyo-ku, Tokyo 112-8551, Japan \\ ${ }^{2}$ Graduate School of Fine Arts, Tokyo University of the Arts, 12-8 Ueno-koen, Taito-ku, Tokyo 110-8714, Japan
}

\begin{abstract}
FePd, FePt, and CoPt alloy epitaxial films of $40 \mathrm{~nm}$ thickness are prepared on $\mathrm{MgO}(111)$ singlecrystal substrates by employing two different methods. One is a one-step method consisting of hightemperature deposition at $600{ }^{\circ} \mathrm{C}$ and the other is a two-step method consisting of low-temperature deposition at $200{ }^{\circ} \mathrm{C}$ followed by annealing at $600{ }^{\circ} \mathrm{C}$. Although the preparation method is different, similar final crystal structures are realized for all the film materials. FePd and FePt alloy films grow on the substrates with six $L 1_{0}(111)$ variants, whose $c$-axes are about $35^{\circ}$ canted from the substrate surface and rotated around the film normal by $60^{\circ}$ each other. The order degrees of FePd and FePt films prepared by one- and two-step methods, $\left(S_{\text {one-step }}, S_{\text {two-step }}\right)$, are estimated to be $(0.25,0.33)$ and $(0.08,0.15)$, respectively. On the contrary, $L 1_{0}$ ordered phase formation is not recognized for CoPt alloy films. The films prepared by one-step method have rough surfaces surrounded by side facets, whereas the films prepared by two-step method have very flat surfaces with the arithmetical mean roughness of $0.3 \mathrm{~nm}$. The two-step method is useful for preparation of $L 1_{0}$ ordered films with flat surface.
\end{abstract}

\section{Introduction}

$L 1_{0}$ ordered FePd, FePt, and CoPt alloys show uniaxial magnetocrystalline anisotropy energies greater than $10^{7}$ $\mathrm{erg} / \mathrm{cm}^{3}$ along the $c$-axis and the thin films have been investigated for magnetic device applications like recording media, etc. Surface flatness is an important technological issue for practical applications. However in order to achieve a high order degree, it is necessary to employ a high temperature processing. Film deposition at a high substrate temperature tends to enhance the film surface roughness due to migration and clustering of deposited atoms [1]. The control of $c$-axis distribution is also required for fabrication of magnetic film devices. In order to investigate the $L 1_{0}$ crystal distribution, a welldefined epitaxial film is useful, since the crystallographic orientation can be controlled by single-crystal substrate. FePd [1-4], FePt [1, 5-8], and CoPt [1, 9-12] epitaxial films have been prepared on $\mathrm{MgO}$ substrates of (001), (110), and (111) orientations. Most of the films have been prepared by employing elevated substrate temperatures around $600{ }^{\circ} \mathrm{C}$.

In our previous studies [13, 14], $L 1_{0}$ ordered FePd, $\mathrm{FePt}$, and CoPt films were prepared on $\mathrm{MgO}$ substrates of (001) and (110) orientations by employing a two-step method; low-temperature deposition at $200{ }^{\circ} \mathrm{C}$ followed by high-temperature annealing at $600{ }^{\circ} \mathrm{C}$. These films had very flat surface with the arithmetical mean roughness
$\left(R_{\mathrm{a}}\right)$ less than $0.3 \mathrm{~nm}$. The films formed on $\mathrm{MgO}(001)$ substrates consisted of $L 1_{0}(001)$ crystal with the $c$-axis normal to the substrate surface and/or $L 1_{0}(100)$ crystal with the $c$-axis lying in the film plane. The films formed on $\mathrm{MgO}(110)$ substrates involved $L 1_{0}(110)$ crystal with the $c$-axis parallel to the substrate surface and $L 1_{0}(011)$ crystal with the $c$-axis $45^{\circ}$ canted from the perpendicular direction. The order degrees of films formed on $\mathrm{MgO}(001)$ and (110) substrates, $\left(S_{\mathrm{FePd}}, S_{\mathrm{FePt}}, S_{\mathrm{CoPt}}\right)$, were $(0.63,0.38,0.16)$ and $(0.29,0.21,0.14)$, respectively. The surface morphology, $c$-axis distribution, and order degree will be influenced by the film orientation, since the migration and clustering of deposited atoms vary depending on the surface free energy of crystallographic plane parallel to the substrate surface. In the present study, the two-step method is applied to preparation of $\mathrm{FePd}$, $\mathrm{FePt}$, and $\mathrm{CoPt}$ films on $\mathrm{MgO}(111)$ substrates. Films are also prepared by using a conventional one-step method consisting of high-temperature deposition at $600{ }^{\circ} \mathrm{C}$. The structural and magnetic properties are compared.

\section{Experimental procedure}

A radio-frequency (RF) magnetron sputtering system equipped with a reflection high-energy electron diffraction (RHEED) facility was employed for film formation. The base pressures were lower than $4 \times 10^{-7} \mathrm{~Pa}$. $\mathrm{FePd}, \mathrm{FePt}$, and CoPt alloy films of $40 \mathrm{~nm}$ thickness were

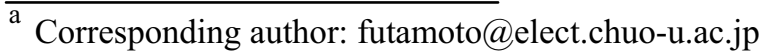


prepared on polished $\mathrm{MgO}(111)$ substrates by the oneand the two-step methods. Before film formation, substrates were heated at $600{ }^{\circ} \mathrm{C}$ for $1 \mathrm{~h}$ in the chamber to obtain clean surfaces. The $R_{\mathrm{a}}$ value estimated by atomic force microscopy was $0.2 \mathrm{~nm}$ (not shown here). $\mathrm{Fe}_{50} \mathrm{Pd}_{50}$, $\mathrm{Fe}_{50} \mathrm{Pt}_{50}$, and $\mathrm{Co}_{50} \mathrm{Pt}_{50}$ (at. \%) alloy targets of 3 in diameter were employed. The Ar gas pressure during sputtering was kept constant at $0.67 \mathrm{~Pa}$. The RF powers for $\mathrm{FePd}$, FePt, and $\mathrm{CoPt}$ targets were respectively fixed at 35,43 , and $45 \mathrm{~W}$, where the deposition rate was 0.02 $\mathrm{nm} / \mathrm{s}$ for all the materials. The film compositions were confirmed by energy dispersive X-ray spectroscopy and the errors were less than 4 at. $\%$ from the target compositions. The surface structure was studied by RHEED. The resulting structure was investigated by $2 \theta / \omega$-scan out-of-plane, $2 \theta \chi / \phi$-scan in-plane, and $\beta$-scan pole-figure XRD with $\mathrm{Cu}-\mathrm{K} \alpha$ radiation $(\lambda=0.15418 \mathrm{~nm})$. The surface morphology was observed by AFM. The magnetization curves were measured by using a vibrating sample magnetometer.

The notations of crystallographic plane and direction are different between disordered $A 1$ and ordered $L 1_{0}$ structures. In the present study, $A 1$-based notation is applied to the $L 1_{0}$ structure for simple comparison with the $A 1$ structure.

\section{Results and discussion}

Figures 1(a) and (b), respectively, show the RHEED patterns observed for FePd films and FePt films prepared on $\mathrm{MgO}(111)$ substrates by employing the one- and the two-step methods. Clear diffraction patterns consisting of streaks are observed for the FePd and the FePt films prepared by both methods. The streaks indicate that the films have atomically flat terraces. The diffraction patterns are in agreement with the schematic diagrams of diffraction pattern simulated for $A 1(111)$ or $L 1_{0}(111)$ surface shown in figure 2. Superlattice reflections are recognized as shown by the arrows in the intensity profiles of figure 1(a) and (b). The crystal structure is thus determined to be $L 1_{0}$. The epitaxial orientation relationship is determined by RHEED as follows,

$$
\begin{array}{lr}
L 1_{0}(111)[1 \overline{1} 0] \| \mathrm{MgO}(111)[1 \overline{1} 0], & \left(\text { type } L 1_{0}-1\right) \\
L 1_{0}(111)[\overline{1} 10] \| \mathrm{MgO}(111)[1 \overline{1} 0], & \left(\text { type } L 1_{0}-2\right) \\
L 1_{0}(111)[\overline{1} 01] \| \mathrm{MgO}(111)[1 \overline{1} 0], & \left(\text { type } L 1_{0}-3\right) \\
L 1_{0}(111)[10 \overline{1}] \| \mathrm{MgO}(111)[1 \overline{1} 0], & \left(\text { type } L 1_{0}-4\right) \\
L 1_{0}(111)[0 \overline{1} 1] \| \mathrm{MgO}(111)[1 \overline{1} 0], & \left(\text { type } L 1_{0}-5\right) \\
L 1_{0}(111)[01 \overline{1}] \| \mathrm{MgO}(111)[1 \overline{1} 0] . & \left(\text { type } L 1_{0}-6\right)
\end{array}
$$

The FePd and the FePt films consist of six $L 1_{0}(111)$ variants whose $c$-axes are about $35^{\circ}$ canted from the substrate surface and rotated around the film normal by $60^{\circ}$ each other. Figure 3 shows the $\beta$-scan pole-figure XRD patterns of FePd films prepared by one- and twostep methods measured by fixing the tile and diffraction angles of $(\alpha, 2 \theta B)$ at $\left(35^{\circ}, 24^{\circ}\right)$, where the scattering vector is $35^{\circ}$ inclined from the in-plane and $L 1_{0}(001)$ superlattice reflection is expected to be detectable. Six reflections which originate from six $L 1_{0}$ variants are recognized with $60^{\circ}$ separation for both films. The pole-

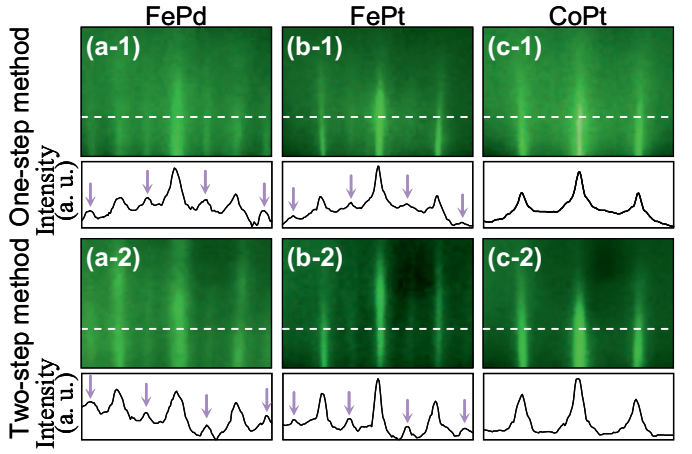

Fig. 1. RHEED patterns and the intensity profiles of (a) FePd, (b) $\mathrm{FePt}$, and (c) CoPt films prepared by (a-1)-(c-1) one- and (a-2)-(c-2) two-step methods. The incident electron beam is parallel to $\mathrm{MgO}[1 \overline{10}]$.

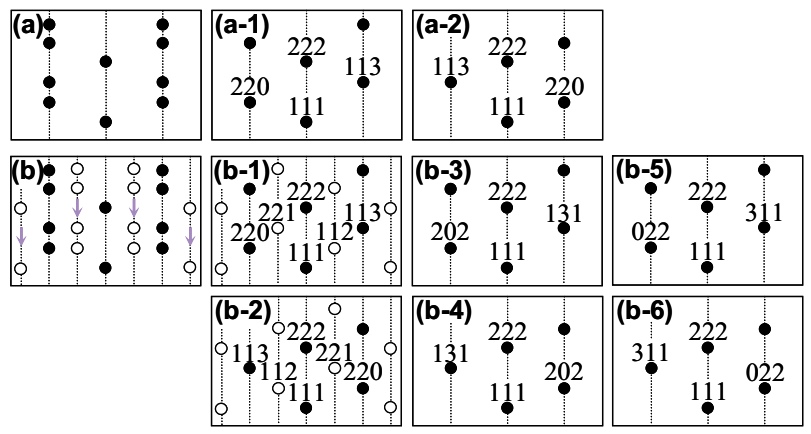

Fig. 2. Schematic diagrams of RHEED patterns simulated for (a) $A 1(111)$ and (b) $L 1_{0}(111)$ surfaces drawn by overlapping (a1) $-(a-2)$ and (b-1)-(b-6), respectively. The diffraction patterns of (a-1)-(a-2) and (b-1)-(b-6) are calculated by using the lattice constants of $a=0.3809 \mathrm{~nm}$ and $(a, c)=(0.3832 \mathrm{~nm}, 0.3773$ $\mathrm{nm})$ The incident electron beam is parallel to $(\mathrm{a}-1, \mathrm{~b}-1)$ [110], (a-

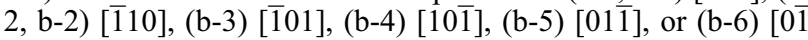
1]. The filled and the open circles respectively correspond to fundamental and superlattice reflections.

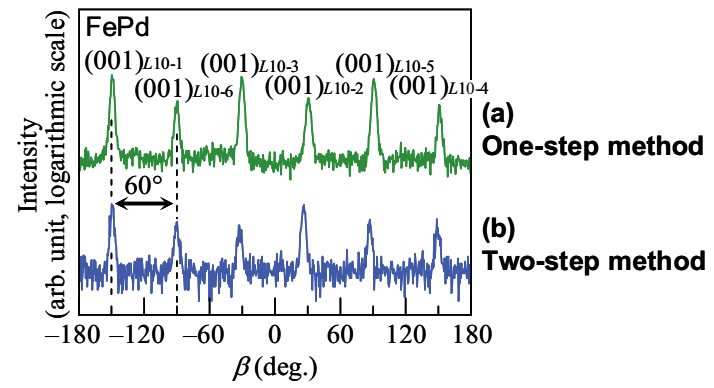

Fig. 3. Pole-figure XRD patterns of FePd films prepared by (a) one- and (b) two-step methods. The angles of $(\alpha, 2 \theta B)$ are fixed at $\left(35^{\circ}, 24^{\circ}\right)$.

figure XRD confirms the crystallographic orientation relationship determined by RHEED.

Figure 1(c-1) and (c-2) show the RHEED patterns observed for CoPt films prepared by one- and two-step methods, respectively. Clear diffraction patterns corresponding to (111) surface are observed and superlattice reflections are absent. The crystal structure and the crystallographic orientation relationship are determined as

$$
\begin{array}{ll}
A 1(111)[1 \overline{1} 0] \| \operatorname{MgO}(111)[1 \overline{1} 0], & \text { (type } A 1-1) \\
A 1(111)[\overline{1} 10] \| \operatorname{MgO}(111)[1 \overline{1} 0] . & \text { (type } A 1-2)
\end{array}
$$




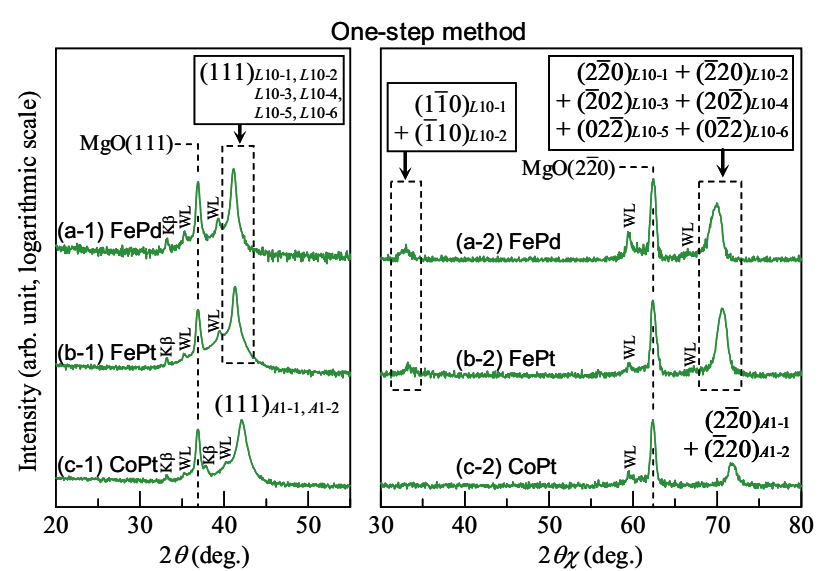

Fig. 4. (a-1)-(c-1) Out-of-plane and (a-2)-(c-2) in-plane XRD patterns of (a) FePd, (b) FePt, and (c) CoPt films prepared by one-step method. The scattering vector of in-plane XRD is parallel to $\mathrm{MgO}[1 \overline{1} 0]$.

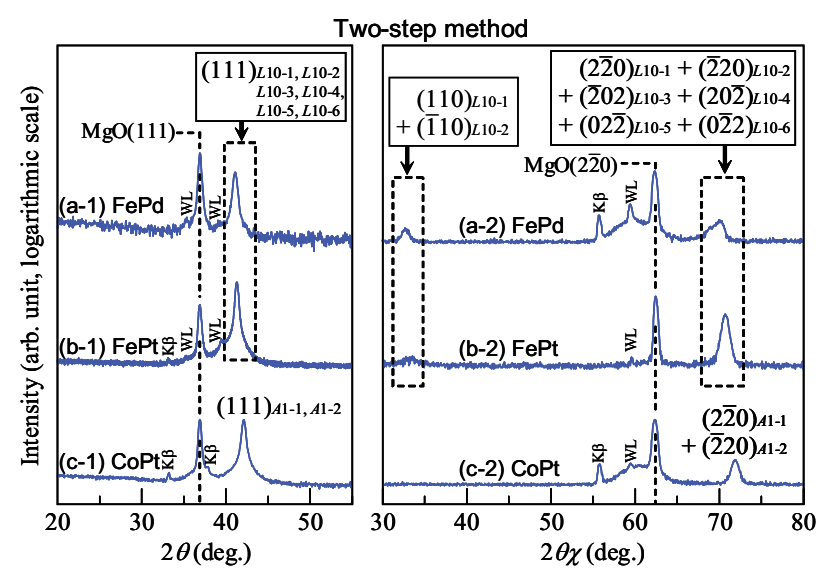

Fig. 5. (a-1)-(c-1) Out-of-plane and (a-2)-(c-2) in-plane XRD patterns of (a) FePd, (b) FePt, and (c) CoPt films prepared by two-step method. The scattering vector of in-plane XRD is parallel to $\mathrm{MgO}[1 \overline{1} 0]$.

The films involve two $A 1(111)$ variants whose atomic stacking sequences of close-packed plane along the perpendicular direction are $\mathrm{ABCABC} \ldots$ and $\mathrm{ACBACB} \ldots$

In ordered to characterize the degree of $L 1_{0}$ ordering $(S)$ by out-of-plane and in-plane XRDs, it is necessary to calculate the structure factors $(F)$ of crystallographic planes expressed as

$$
\begin{aligned}
F_{(h k l)} & =\left\{\frac{1+S}{2} f_{\text {Fe or Co }}+\frac{1-S}{2} f \text { Pd or Pt }\right\}\left\{1+e^{2 \pi i\left(\frac{1}{2} x j+\frac{1}{2} y j\right)}\right\} \\
& +\left\{\frac{1+S}{2} f_{\mathrm{Pd} \mathrm{orPt}}+\frac{1-S}{2} f_{\mathrm{Fe} \mathrm{or} \mathrm{Co}}\right\}\left\{e^{2 \pi i\left(\frac{1}{2} x j+\frac{1}{2} z j\right)}+e^{2 \pi i\left(\frac{1}{2} y j+\frac{1}{2} z j\right)}\right\} .
\end{aligned}
$$

Thus, $F_{(111)}=F_{(2 \overline{2} 0)}=F_{(\overline{2} 20)}=F_{(\overline{2} 02)}=F_{(20 \overline{2})}=F_{(02 \overline{2})}=F_{(0 \overline{2} 2)}=$ $2\left(f_{\mathrm{Pd} \text { or Pt }}+f_{\mathrm{Fe} \text { or } \mathrm{Co}}\right), F_{(\overline{101})}=F_{(10 \overline{1})}=2 S\left(f_{\mathrm{Pd} \text { or Pt }}-f_{\mathrm{Fe} \text { or } \mathrm{Co}}\right)$, $F_{(\overline{1} 01)}=F_{(10 \overline{1})}=F_{(01 \overline{1})}=F_{(\overline{1} 10)}=0$. The reflections from (111), $(2 \overline{2} 0),(\overline{2} 20),(\overline{2} 02),(20 \overline{2}),(02 \overline{2})$, and $(0 \overline{2} 2)$, from $(1 \overline{1} 0)$ and $(\overline{1} 10)$, from $(\overline{101}),(10 \overline{1}),(01 \overline{1})$, and $(0 \overline{1} 1)$ are fundamental, superlattice, and forbidden, respectively.

Figures $4(a, b)$ and $5(a, b)$ show the out-of-plane and in-plane XRD patterns of FePd and FePt films prepared by one- and two-step methods. Fundamental (111) and $(2 \overline{2} 0)+(\overline{2} 20)+(\overline{2} 02)+(20 \overline{2})+(02 \overline{2})+(0 \overline{2} 2)$ reflections are observed in the out-of-plane and the in-plane patterns, respectively. Superlattice $(1 \overline{1} 0)+(\overline{1} 10)$ reflections are recognized around $33^{\circ}$ in the in-plane patterns.

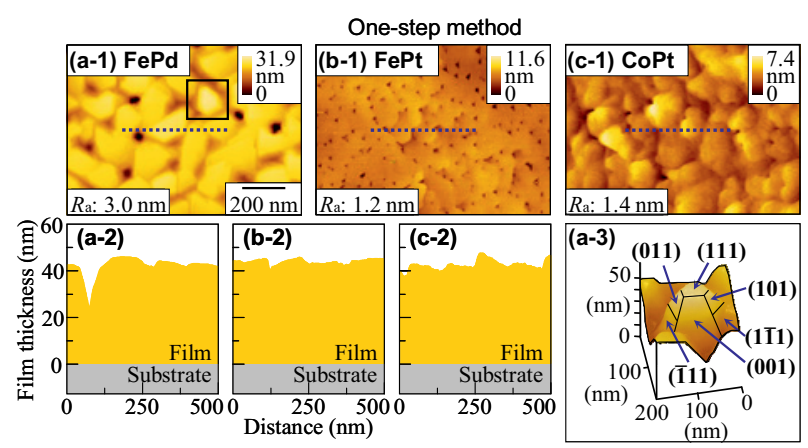

Fig. 6. (a-1)-(c-1) AFM images observed for (a-1) FePd, (b-1) FePt, and (c-1) CoPt films prepared by one-step method. (a-2)(c-2) Cross-sectional profiles measured along the dotted line in (a-1)-(c-1), respectively. (a-3) Three dimensional image of the area surround by black lines in (a-1).
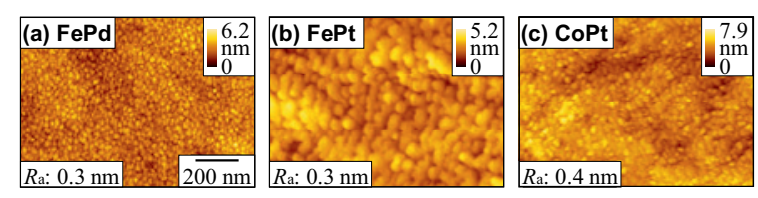

Fig. 7. (a)-(c) AFM images observed for (a) FePd, (b) FePt and (c) CoPt films deposited on $\mathrm{MgO}(111)$ substrates at $200{ }^{\circ} \mathrm{C}$.

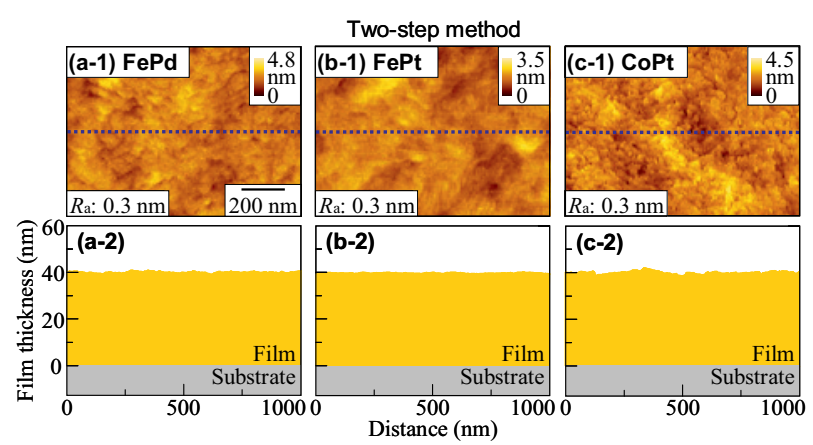

Fig. 8. (a-1)-(c-1) AFM images observed for (a-1) FePd, (b-1) FePt, and (c-1) CoPt films prepared by two-step method. (a-2)(c-2) Cross-sectional profiles measured along the dotted line in (a-1)-(c-1), respectively.

The $S$ is calculated from the in-plane XRD data. The XRD integrated intensity $(I)$ is proportional to $\left|F F^{*}\right|$, Lorentz-polarization factor $(L)$, and absorption factor $(A)$ [15]. In the present paper, an influence of temperature factor, which is often omitted when comparing intensities of two reflections, is not considered. The intensity ratio of $I_{\mathrm{s}} / I_{\mathrm{f}}=\left(I_{(1 \overline{1} 0)}+I_{(\overline{1} 10)}\right) /\left(I_{(2 \overline{2} 0)}+I_{(\overline{2} 20)}+I_{(\overline{2} 02)}+I_{(20 \overline{2})}+I_{(02 \overline{2})}+I_{(0 \overline{2} 2)}\right)=$ $\left[2\left\{2 S\left(f_{\mathrm{Pd} \text { or Pt }}-f_{\mathrm{Fe}}\right)\right\}^{2} L_{\mathrm{s}} A_{\mathrm{s}}\right] /\left[6\left\{2\left(f_{\mathrm{Pd} \text { or Pt }}+f_{\mathrm{Fe}}\right)\right\}^{2} L_{\mathrm{f}} A_{\mathrm{f}}\right]$. Therefore, the $S$ is expressed as

$$
S=\left[\left(I_{\mathrm{s}} / I_{\mathrm{f}}\right) \times 3\left\{\left(f_{\mathrm{Fe}}+f_{\mathrm{Pd} \text { or Pt }}\right) /\left(f_{\mathrm{Fe}}-f_{\mathrm{Pd} \text { or Pt }}\right)\right\}^{2} \times\left(L_{\mathrm{f}} A_{\mathrm{f}} / L_{\mathrm{s}} A_{\mathrm{s}}\right)\right]^{1 / 2} .
$$

The order degrees of FePd and FePt films prepared by oneand two-step methods, $\left(S_{\text {one-step }}, S_{\text {two-step }}\right)$, are calculated to be $(0.25,0.33)$ and $(0.08,0.15)$, respectively. Although the preparation method is different, similar order degrees are observed for the respective film materials.

Figures 4(c) and 5(c) show the XRD patterns of $\mathrm{CoPt}$ films prepared by one- and two-step methods, respectively. Only fundamental reflections are observed for both films.

Figure 6(a-1)-(c-1) and (a-2)-(c-2) show the AFM images and the cross-sectional profiles of films prepared by one-step method, respectively. Three-dimensional 
island nucleation occurs and island-like surfaces involving side facets are observed. The orientations of facets are estimated from the three-dimensional AFM data shown, for example, in figure $6(\mathrm{a}-3)$ to be $\{111\}$, $\{001\}$, and $\{011\}$ which have low surface free energies in the $L 1_{0}$ structure. The $R_{\mathrm{a}}$ values of FePd, FePt, and CoPt films are 3.0, 1.2, and $1.4 \mathrm{~nm}$, respectively. Figure 7 shows the AFM images observed for FePd, FePt, and CoPt films deposited at $200{ }^{\circ} \mathrm{C}$. Figure 8 shows the AFM data of films deposited at $200{ }^{\circ} \mathrm{C}$ followed by annealing at $600{ }^{\circ} \mathrm{C}$, that is, films prepared by two-step method. The $R_{\mathrm{a}}$ values of films before and after annealing are below $0.4 \mathrm{~nm}$ and $0.3 \mathrm{~nm}$, respectively. It is clearly shown that a very flat surface is obtained by employing the two-step method.

Figure 9 shows the magnetization curves. The hysteresis curves are almost isotropic in the in-plane measurements for all the films. The easy magnetization axis of $L 1_{0}(111)$ film does not exist in the film plane, since the $c$-axis is $35^{\circ}$ inclined from the in-plane. In addition, a complex $L 1_{0}$ variant structure is formed in the film where the easy magnetization axes, [001], of six variant crystals are coexisting. This could be the reason why almost isotropic in-plane magnetization curves are observed for these films.

\section{Conclusion}

FePd, FePt, and CoPt alloy epitaxial films of $40 \mathrm{~nm}$ thickness are prepared on $\mathrm{MgO}(111)$ substrates by employing two different methods; one-step method consisting of deposition at $600{ }^{\circ} \mathrm{C}$ and two-step method consisting of deposition at $200{ }^{\circ} \mathrm{C}$ followed by annealing at $600{ }^{\circ} \mathrm{C}$. Similar final crystal structures, order degrees, and magnetic properties are observed in the films prepared by both methods for respective film materials. FePd and FePt films consist of six $L 1_{0}(111)$ variants with the $c$-axis $35^{\circ}$ canted from the substrate surface, whereas ordered phase formation is not recognized in CoPt films. CoPt films consist of two A1(111) variants. The order degrees, $\left(S_{\text {one-step }}, S_{\text {two-step }}\right)$, of FePd and FePt films are $(0.25,0.33)$ and $(0.08,0.15)$, respectively. These films show in-plane magnetic properties. However, the surface flatness is quite different between the two cases. The films prepared by two-step method have very flat surfaces with the $R_{\mathrm{a}}$ value lower than $0.3 \mathrm{~nm}$, whereas the film prepared by the one-step method consist of islandlike surfaces involving side facets. The two-step method is found to be useful for preparations of very flat thin film with $L 1_{0}$ ordered structure.

\section{Acknowledgements}

This work was supported by JSPS KAKENHI Grant Number 25420294, JST A-STEP Grant Number AS242Z00169M, and Chuo University Grant for Special Research.

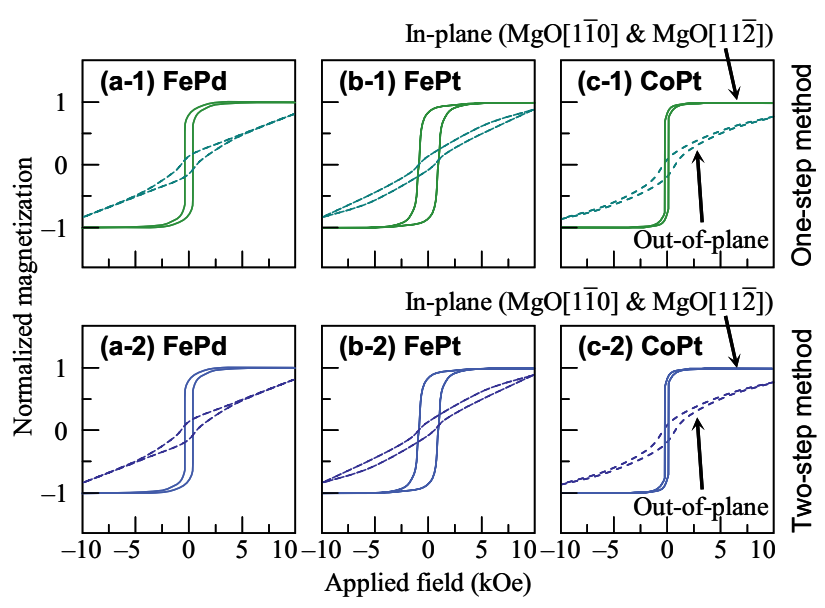

Fig. 9. Magnetization curves of (a) FePd, (b) FePt, and (c) CoPt films prepared by (a-1)-(c-1) one- and (a-2)-(c-2) twostep methods.

\section{References}

1. M. Ohtake, S. Ouchi, F. Kirino, M. Futamoto, J. Appl. Phys. 111, 07A708 (2012)

2. V. Gehanno, A. Marty, B. Gilles, Y. Samson, Phys. Rev. B 55, 12552 (1997)

3. P. R. Aitchison, J. N. Chapman, V. Gehanno, I. S. Weir, M. R. Scheinfein, S. McVitie, A. Marty, J. Magn. Magn. Mater. 223, 138 (2001)

4. A. Cebollada, P. Caro, J. L. Menéndez, F. Briones, D. García, A. Hernando, and J. A. García Díaz, J. Magn. Magn. Mater. 203, 162 (1999)

5. B. M. Lairson, M. R. Visokay, R. Sinclair, B. M. Clemens, Appl. Phys. Lett. 62, 639 (1993)

6. A. Cebollada, D. Weller, J. Sticht, G. R. Harp, R. F. C. Farrow, R. F. Marks, R. Savoy, J. C. Scott, Phys. Rev. B 50, 3419 (1994)

7. R. F. C. Farrow, D. Weller, R. F. Marks, M. F. Toney, D. J. Smith, M. R. McCartney, J. Appl. Phys. 84, 934 (1998)

8. P. Mani, V. V. Krishnamurthy, S. Maat, A. J. Kellock, J. L. Robertson, G. J. Mankey: J. Vac. Sci. Technol. A 23, 785 (2005)

9. M. R. Visokay, R. Sinclair, Appl. Phys. Lett. 66, 1692 (1995)

10. O. Ersen, V. Parasote, V. Pierron-Bohnes, M. C. Cadeville, C. Ulhaq-Bouillet, J. Appl. Phys. 93, 2987 (2003)

11. M. Abes, O. Ersen, C. Meny, G. Schmerber, M. Acosta, J. Arabski, C. Ulhaq-Bouillet, A. Dinia, P. Panissod, V. Pierron-Bohnes, J. Appl. Phys. 101, 063911 (2007)

12. A. C. Sun, F. T. Yuan, J. H. Hsu, H.Y. Lee, Scr. Mater. 61, 713 (2009)

13. A. Itabashi, M. Ohtake, S. Ouchi, F. Kirino, M. Futamoto, EPJ Web Conf. 40, 07001 (2013)

14. M. Ohtake, A. Itabashi, F. Kirino, M. Futamoto, IEEE Trans. Magn. 49, 3295 (2013)

15. B. D. Cullity, in Elements of X-Ray Diffraction (Addsion-Wesley, Massachusetts, 1956) pp. 104-137 